\title{
Inhibitory Effect of Cortisol on the Defense Activities of Tilapia Neutrophils in Vitro
}

\author{
Junko Kurogi ${ }^{1}$ and Takaji lida ${ }^{2 *}$ \\ ${ }^{1}$ The United Graduate School of Agricultural Sciences, Kagoshima University, Korimoto 1-21-24, \\ Kagoshima 890-0065, Japan \\ ${ }^{2}$ Faculty of Agriculture, Miyazaki University, Gakuen Kibanadai-nishi 1-1, \\ Miyazaki 889-2192, Japan.
}

(Received July 31, 2001)

\begin{abstract}
On the premise that fish neutrophil activities are depressed under stressed conditions, this study investigated the direct effect of cortisol on the in vitro activity of neutrophiles collected from tilapia Oreochromis niloticus. Neutrophils were obtained from swim bladders and exposed to different levels of cortisol $(0-1000 \mathrm{ng} / \mathrm{mL})$, and their chemotactic, phagocytic and respiratory burst activities were assessed. This treatment suppressed neutrophil activities as seen in the reduction of chemotactic, phagocytic and respiratory burst activities. Neutrophil activities appeared to be suppressed in a dose-dependent manner leading to a considerable reduction in neutrophil activities with high concentrations of cortisol. Nevertheless, we showed that the respiratory burst activity was able to recover from the observed effect after cortisol was removed. The results in this study strongly suggest that cortisol has a directly adverse effect on fish neutrophil defense activities, but that the effect is reversible.
\end{abstract}

Key words: cortisol, neutrophil activity, Oreochromis niloticus, tilapia, stress

Stress has been well documented for its immunosuppressive actions. In teleost fish, immunological changes associated with stress have been studied for almost twenty years (Ellis, 1981). Corticosteroids, produced by the activation of HPI (hypothalamus-pituitaryinterrenal) axis which stress stimulates, are considered to be important mediators of the immune response (Balm, 1997; Sumpter, 1997). Cortisol is released from interrenal tissue after the stimulation of higher brain centers in fish (Balm, 1997) and is widely recognized as an immuno-suppressive agent both in mammals (Oda and Katori, 1992) and in fish.

Kurogi and lida (1999) demonstrated that social hierarchy in tilapia induced suppression of neutrophil defense activities in subordinate fish and that cortisol concentrations in blood plasma of the subordinate fish were significantly higher than dominant fish. Much attention has been paid to the interactions between cortisol and fish lymphocytes (Tripp et al., 1987). However, little is known whether cortisol influences neutrophil activity, even though neutrophils play an important role in the first stages of infection. In this study, we aimed to reveal

\footnotetext{
* Corresponding author

E-mail: takaji@cc.miyazaki-u.ac.jp
}

the effect of cortisol on neutrophil defense activities in vitro by means of analysing their chemotactic, phagocytic and respiratory burst activities.

\section{Materials and Methods}

Fish

Tilapia (Oreochromis niloticus) of $76 \mathrm{~g}$ mean weight (hatched and raised in our laboratory at Miyazaki University) were used in this study. The fish were maintained for at least $3 \mathrm{wk}$ in $150 \mathrm{~L}$ plastic tanks containing constantly aerated water at $25^{\circ} \mathrm{C}$. They were fed daily with a commercial diet.

\section{Preparation of cortisol solution}

Cortisol (hydrocortisone; Wako) stock solution was prepared according to the method described by Tripp et al. (1987). Briefly, the stock solution was prepared by dissolving an appropriate quantity of cortisol in $95 \%$ ethanol, allowing the ethanol to completely evaporate, and re-suspending the residual cortisol in Hanks' balanced salt solution without phenol red (HBSS). The solution was filter-sterilized and the final concentration was measured using a commercial kit (Enzaplate cortisol; Chiba Corning Diagnostics Inc.) prior to use. 
Neutrophil preparation

Neutrophils used in this experiment were collected from the tilapia swim bladders. The collecting procedure was in accordance with the method described previously by Endo et al. (1997). Collected neutrophils were washed twice by centrifugation at $200 \times g$ for $5 \mathrm{~min}$ and re-suspended in HBSS.

\section{Neutrophil activities}

Chemotactic activity was estimated by using a modified Boyden chamber, for $3 \mathrm{~h}$, according to Nagamura and Wakabayashi (1985). Zymosan-treated normal tilapia sera were used as a chemotactic factor. The neutrophils in the filter were stained with hematoxylin, and the number of cells migrating more than $80 \mu \mathrm{m}$ were counted under a microscope.

Phagocytic activity for $1 \mathrm{~h}$ against opsonized zymosan, calculated as index of phagocytosis (Le Morvan et al., 1997), and respiratory burst activity for $10 \mathrm{~min}$, measured as CLA-enhanced chemiluminescent response, were assessed as previously reported (Kurogi and lida, 1999).

\section{Cortisol treatment}

The neutrophil density was adjusted to $1 \times 10^{6}$ cells/ $\mathrm{mL}$ with HBSS containing different concentrations of cortisol $(0,1,10,100,1000 \mathrm{ng} / \mathrm{mL})$ and incubated at $25^{\circ} \mathrm{C}$ for $1 \mathrm{~h}$ (Stave and Robertson, 1985). After the incubation, the defense activities of the neutrophils were estimated. Because cortisol was contained in the neutrophil suspensions during the measurements of the activities, total cortisol treatments were for $4 \mathrm{~h}, 2 \mathrm{~h}$ or 70 min for the chemotactic, phagocytic or respiratory burst activities, respectively. These assessments were repeated 5 times each.

Restoration of neutrophil respiratory burst activity from the effect of cortisol was determined by removal of cortisol, $1000 \mathrm{ng} / \mathrm{mL}$, from the mixture. The cortisol solution was removed from the mixtures by centrifuging at $200 \times g$ for 5 min twice after $1 \mathrm{~h}$ incubation. Washed neutrophils were then divided into 2 parts; half was replaced with cortisol solution $(1000 \mathrm{ng} / \mathrm{mL})$ and the other was put in HBSS. The activity was immediately assessed. The experiment was repeated 5 times.

\section{Statistic analysis}

All data were analysed by Student's $t$-test and significant differences were determined at $\mathrm{P}<0.05$.

\section{Results}

\section{Neutrophil activities}

Chemotaxis of neutrophils was depressed by cortisol in a dose-dependent manner as seen in Fig. 1. Percentage of the neutrophils migrating more than $80 \mu \mathrm{m}$ in cortisol concentrations of 100 and $1000 \mathrm{ng} / \mathrm{mL}$ were

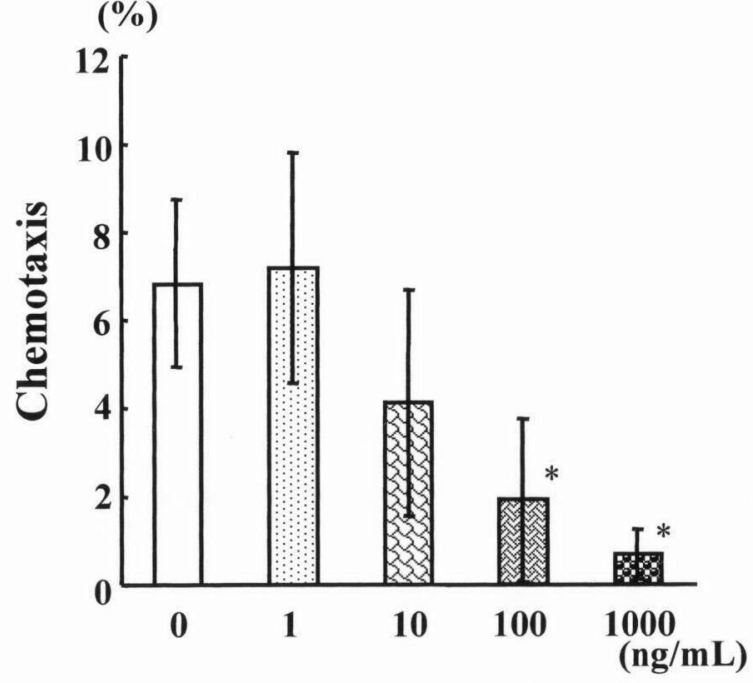

\section{Cortisol concentration}

Fig. 1. Effect of cortisol concentrations on chemotactic activity of tilapia neutrophils in vitro. Chemotaxis expressed as percentage of the neutrophils migrating more than $80 \mu \mathrm{m}$. Bars with asterisks are significantly different from 0 , control $(p<0.05) . \quad n=5$.

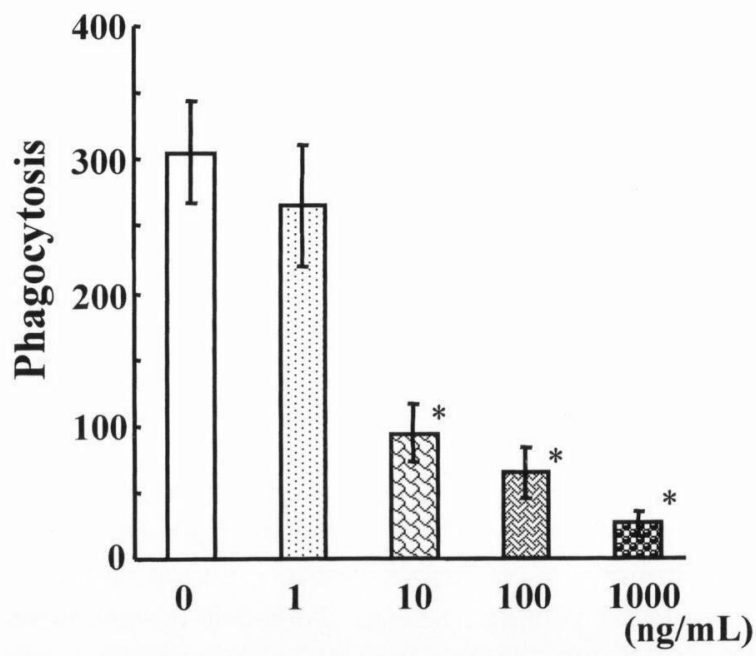

Cortisol concentration

Fig. 2. Effect of cortisol concentrations on phagocytic activity of tilapia neutrophils in vitro. Phagocytosis calculated as index of phagocytosis. Bars with asterisks are significantly different from 0 , control $(p<0.05) . \quad n=5$.

$1.9 \pm 1.8$ and $0.7 \pm 0.6$, respectively, with significant decreases compared with the control value $(6.8 \pm 1.9 \%)$.

Reduced phagocytosis and respiratory burst of the neutrophils in cortisol solution were observed as seen in Figs. 2 and 3 respectively. These reductions by cortisol were also detected dose-dependently. At more than 10 $\mathrm{ng} / \mathrm{mL}$, phagocytosis was suppressed significantly from control, and at more than $100 \mathrm{ng} / \mathrm{mL}$, respiratory burst was repressed significantly from control. 


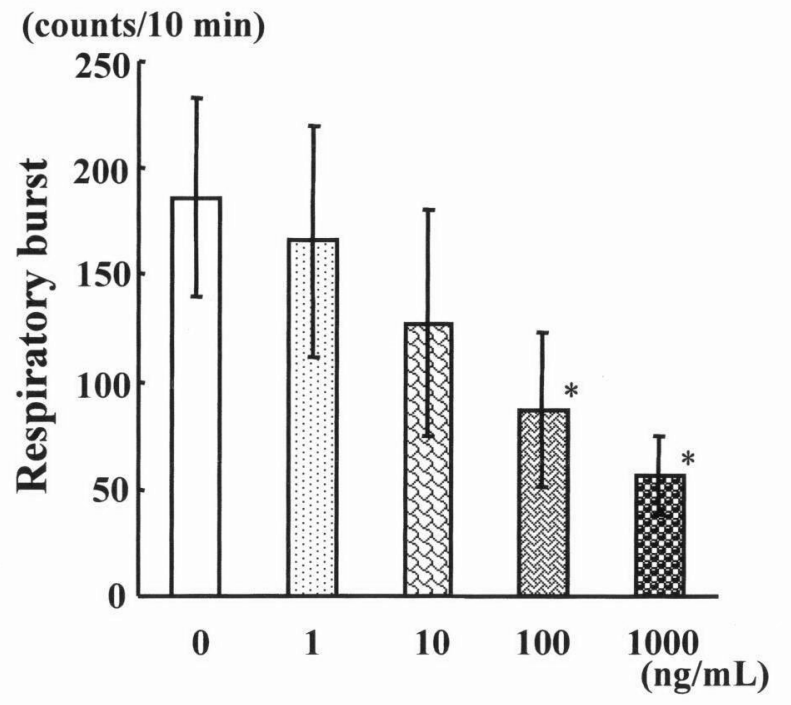

Cortisol concentration

Fig. 3. Effect of cortisol concentrations on respiratory burst of tilapia neutrophils in vitro. Respiratory burst expressed as chemiluminescent response (counts/10 $\mathrm{min})$. Bars with asterisks are significantly different from 0 , control $(p<0.05) . \quad n=5$.

\section{Recovery from the effect of cortisol}

One hour after incubating neutrophils in HBSS, the respiratory burst activity of neutrophils slightly decreased, without significant difference from the activity before incubation. However, incubation for $1 \mathrm{~h}$ in cortisol solution (1000 $\mathrm{ng} / \mathrm{mL}$ ) significantly reduced the activity. On the other hand, remove of cortisol after $1 \mathrm{~h}$ incubation in the cortisol solution almost completely restored the activity (Table 1 ).

Table 1. Restoration of respiratory burst of tilapia neutrophils from adverse effect of cortisol in vitro

\begin{tabular}{lcc}
\hline & \multicolumn{2}{c}{ Respiratory burst (counts/10 min) } \\
\cline { 2 - 3 } Culture solution & \multicolumn{2}{c}{$\begin{array}{l}\text { Incubation time (min) } \\
0^{\text {b) }}\end{array}$} \\
\hline Control (HBSS) & 60 \\
Cortisol $^{\text {d })}$ & $297.4 \pm 70.5$ & $241.2 \pm 18.9$ \\
Cortisol-HBSS $^{\text {d) }}$ & $198.8 \pm 57.3^{*}$ & $126.7 \pm 83.2^{*}$ \\
\hline${ }^{\text {a) }}$ Respiratory burst was measured as chemiluminescence \\
for 10 min (counts/10 min). \\
b) The neutrophils were assessed their ability in HBSS or \\
cortisol solution immediately after the collection. \\
c) Control neutrophils were incubated with HBSS for 60 min. \\
d) After neutrophils were incubated with cortisol solution for \\
60 min, neutrophils washed with HBSS by centrifugation \\
were then divided into 2 parts; half was replaced with cor- \\
tisol solution (Cortisol) and the other was put in HBSS \\
(Cortisol-HBSS). \\
Asterisks indicate significant differences from control. \\
$\mathrm{n}=5$
\end{tabular}

\section{Discussion}

The immunological changes associated with the response of fish to stress have received considerable attention since the early 1980s (Ellis, 1981). The stimulation of higher brain center triggered by stress evokes HPI (hypothalamo-pituitary-interrenal) axis actions and produces many kinds of hormones such as corticotropin releasing hormone $(\mathrm{CRH})$, adrenocorticotropic hormone $(\mathrm{ACTH})$, catecholamine and cortisol through the stimulation processes reviewed by Weyts et al. (1999).

Recently research has focused upon the effect of "stress hormones" on fish defense mechanisms. In channel catfish Ictalurus punctatus, Ellsaesser and Clem (1987) showed cortisol treatment in vivo led to lymphopenia and neutrophilia. Nagae et al. (1994) demonstrated that cortisol suppressed plasma immunogloblin $\mathrm{M}$ in masu salmon Oncorhynchus masou. Reduced generation of antibody-producing cells in coho salmon $O$. kisutch has also been attributed to cortisol (Maule et al., 1987). A considerable amount of evidence suggests that physiological consequences through the activation of the $\mathrm{HPI}$ are as a direct result of elevated cortisol concentrations that occur in response to stress. Moreover, previous in vitro studies showed the inhibition of chemiluminescence response of phagocytes in striped bass Morone saxatilis (Stave and Robertson, 1985), reduced B cell activation in coho salmon (Tripp et al., 1987) and suppressed nitric oxide production in goldfish Carassius auratus macrophages by cortisol (Wang and Belosevic, 1995).

The results of this study show that cortisol has drastic dose-dependent effects on fish neutrophil defense activities in vitro, which can be seen as a clear reduction in their chemotactic, phagocytic and respiratory burst activities at high doses. Concentrations less than $1 \mathrm{ng} /$ $\mathrm{mL}(1-1000 \mathrm{pg} / \mathrm{mL})$ indicated neither an increase nor decrease in the activities compared to control of $0 \mathrm{ng} / \mathrm{mL}$ (data not shown). Our findings suggest that increased plasma cortisol in fish would depress neutrophil defense mechanisms. Previously, we identified impaired neutrophil defense activities in socially stressed fish (Kurogi and lida, 1999), where the cortisol concentration of the stressed fish was about $20 \mathrm{ng} / \mathrm{mL}$ in plasma. The down-regulation of the activities in that study was most likely due to the direct effect of cortisol. In addition, we suggest that the neutrophil activity can be restored once cortisol is eliminated. This outcome is in accordance with a similar report in humans (Dandona et al., 1999).

Some studies have indicated that glucocorticoids depressed porcine neutrophil activities such as migration and chemiluminescence in vitro (Salak et al., 1993), and respiratory burst of human neutrophils were also depressed by cortisol (Umeki and Soejima, 1990). However, neutrohils are not always adversely affected by cortisol. In fact, in vitro chemotaxis of cattle neutro- 
phils was increased by dexamethasone (Anderson et al., 1999). In teleost, Weyts et al. (1998) have shown that respiratory burst activity of carp neutrophils was not influenced by cortisol in vitro or in vivo. They used cortisol at less than $10^{-6} \mathrm{M}(3.6 \mathrm{ng} / \mathrm{mL})$. In the present study, cortisol (more than $10 \mathrm{ng} / \mathrm{mL}$ ) obviously suppressed the defense activities of tilapia neutrophils, but it is still not clear how cortisol affects the neutrophil activities. The results from some studies have shown inconsistency about the mechanisms involved (Lippman and Barr, 1977; Fantuzzi and Ghezzi, 1993; Weyts et al., 1998). It is possible that cortisol can adversely affect neutrophils in several ways.

A few other studies have reported that fish with elevated plasma cortisol had a significantly higher susceptibility to infectious diseases (Pickering and Duston, 1983; Pickering and Pottinger, 1985). They showed that there was no association with reduced lymphocytes in causing diseases, meaning that causing diseases relates to cortisol rather than number of circulating lymphocytes. Cortisol and fish defense mechanisms still have complex inter-relationships. Therefore it remains for further in vivo study to reveal this relationship.

\section{Acknowledgement}

Our sincere thanks go to Mr. Carl Gough, Department of Zoology, University of Aberdeen, U. K., for brushing the manuscript up and his critical comments on it.

\section{References}

Anderson, B. H., D. L. Watson and I. G. Colditz (1999): The effect of dexamethasone on some immunological parameters in cattle. Vet. Res. Commun., 23, 399-413.

Balm, P. H. M., (1997): Immune-endocrine interactions, In "Fish stress and health in aquaculture" (ed. by G. K. Iwama, A. D. Pickering, J. P. Sumpter and C. B. Schreck). Cambridge University press, Cambridge, pp. 195-221.

Dandona, P., M. Suri, W. Hamouda, A. Aljada, Y. Kumbkari and K. Thusu (1999): Hydrocortisone-induced inhibition of reactive oxygen species by polymorphonuclear neutrophils. Crit. Care. Med., 27, 2442-2444.

Ellis, A. E. (1981): Stress and the modulation of defense mechanisms in fish, In "Stress and fish" (ed. by A. D. Pickering). Academic press, London, pp. 147-170.

Ellsaesser, C. F. and L. W. Clem (1987): Cortisol-induced hematologic and immunologic changes in channel catfish (Ictalurus punctatus). Comp. Biochem. Physiol., 87A, 405-408.

Endo,M., C. Arunlertaree, L. Ruangpan, A. Ponpornpisit, T. Yoshida and T. lida (1997): A new method for collecting neutrophils using swim bladder. Fisheries Sci., 63, 644645.

Fantuzzi, G. and P. Ghezzi (1993): Glucocorticoids as cytokine inhibitors: role in neuroendocrine control and therapy of inflammatory diseases. Med. Inflam, 2, 263-270.
Kurogi, J. and T. lida (1999): Social stress suppressed defense activities of neutrophils in tilapia. Fish Pathol., 34, 15-18.

Le Morvan, C., P. Clerton, P. Deschaux and D. Troutaud (1997): Effects of environmental temperature on macrophage activities in carp. Fish Shellfish Immunol., 7, 209212.

Lippman, M. and R. Barr (1977): Glucocorticoid receptors in purified subpopulations of human peripheral blood lymphocytes. J. Immunol., 118, 1977-1981.

Maule, A. G., C. B. Schreck and S. L. Kaattari (1987): Changes in the immune system of coho salmon (Oncorhyncus kisutch) during the parr-to-smolt transformation and after implantation of cortisol. Can. J. Fish. Aquat. Sci., 44, 161-166.

Nagae, M., H. Fukuda, K. Ura, H. Kawamura, S. Adachi, A. Hara and K. Yamauchi (1994): The effect of cortisol administration on blood plasma immunogloblin $\mathrm{M}$ (IgM) concentrations in masu salmon (Oncorhynchus masou). Physiol. Biochem., 13, 41-48.

Nagamura, Y. and H. Wakabayashi (1985): Changes in glycogen content of neutrophils in eel, Anguilla japonica by bacterial infection. Fish Pathol., 20, 389-394.

Oda, T. and M. Katori (1992): Inhibition site of dexamethason on extravasation of polymorphonuclear leukocytes in the hamster cheek pouch microcirculation. J. Leu. Biol., 52, 337-342.

Pickering, A. D. and J. Duston (1983): Administration of cortisol to brown trout, Salmo trutta L., and its effects on the susceptibility to Saprolegnia infection and furunculosis. J. Fish Biol., 23, 163-175.

Pickering, A. D. and T. G. Pottinger (1985): Cortisol can increase the susceptibility of brown trout, Salmo trutta L., to disease without reducing the white blood cell count. $J$. Fish Biol., 27, 611-619.

Salak, J. L., J. J. McGlone, M. Lyte (1993): Effects of in vitro adrenocorticotrophic hormone, cortisol and human recombinant interleukin-2 on porcine neutrophil migration and luminol-dependent chemiluminescence. Vet. Immunol. Immunopathol., 39, 327-337.

Stave, J. W. and B. S. Robertson (1985): Hydrocortisone suppresses the chemiluminescent response of striped bass phagocytes. Dev. Comp. Immunol., 9, 77-84.

Sumpter, J. P. (1997): The endocrinology of stress, In "Fish stress and health in aquaculture" (ed. by G. K. Iwama, A.D. Pickering, J. P. Sumpter and C. B. Schreck). Cambridge University Press, Cambridge, pp. 98-118.

Tripp, R. A., A. G. Maule, C. B. Schrecl and S. L. Kaattari (1987): Cortisol mediated suppression of salmonid lymphocyte response in vitro. Dev. Comp. Immunol., 11, 565576.

Umeki, S. and R. Soejima (1990): Hydrocortisone inhibits the respiratory burst oxidase from human neutrophils in wholecell and cell-free systems. Biochem. Biophys. Acta., 1052, 211-215.

Wang, R. and M. Belosevic (1995): The in vitro effects of oestradiol and cortisol on the function of a long-term goldfish macrophage cell line. Dev. Comp. Immunol., 19, 327-336.

Weyts, F. A. A., G. Flik and B. M. L. Verburg-Van Kemenade (1998): Cortisol inhibits apoptosis in carp neutrophilic granulocytes. Dev. Comp. Immunol., 22, 563-572.

Weyts, F. A. A., N. Cohen, G. Flik and B. M. L. Verburg-Van Kemenade (1999): Interactions between the immune system and the hypothalamo-pituitary-interrenal axis in fish. Fish Shelfish Immunol., 9, 1-20. 